Supplement of Biogeosciences, 16, 3777-3792, 2019

https://doi.org/10.5194/bg-16-3777-2019-supplement

(C) Author(s) 2019. This work is distributed under

the Creative Commons Attribution 4.0 License.

(c) (1)

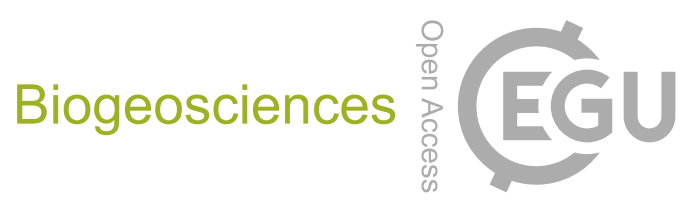

Supplement of

\title{
Seasonal and spatial patterns of primary production in a high-latitude fjord affected by Greenland Ice Sheet run-off
}

\section{Johnna M. Holding et al.}

Correspondence to: Johnna M. Holding (johnna@bios.au.dk)

The copyright of individual parts of the supplement might differ from the CC BY 4.0 License. 
Supplementary Material for:

"Seasonal and spatial patterns of primary production in a high-latitude fjord affected by Greenland Ice Sheet run-off"- J.M. Holding et al.

Table S1. Photosynthetic parameters for each PI curve performed. PI curves were performed on each sampling day at $2-3$ depths per station (surface and depth(s) of florescence maxiumum). $\mathrm{P}^{\mathrm{B}} \mathrm{m}_{\mathrm{m}}$ the chlorophyll standardized maximum carbon uptake ( $\mathrm{g} \mathrm{C}$ $\left.\mathrm{g}^{-1} \mathrm{Chl} \mathrm{h}^{-1}\right) \cdot \mathrm{a}_{\mathrm{B}}=$ the light utilization efficiency value standardized for chlorophyll $\left(\mathrm{g} \mathrm{C} \mathrm{g}^{-1} \mathrm{Chl}\right.$ mol-1 $^{-1}$ photons $\left.\mathrm{m}^{2}\right)$. Ik- values $\left(\mathrm{P}_{\mathrm{m}}^{\mathrm{B}} / \mathrm{a}_{\mathrm{B}}\right)=$ the light level that is saturating for carbon fixation $\left(\mu \mathrm{mol}\right.$ photons $\left.\mathrm{m}^{-2} \mathrm{~s}^{-1}\right)$.

\begin{tabular}{|c|c|c|c|c|c|}
\hline Date & Julian Day & Depth (m) & $\mathbf{P}_{\mathrm{m}}^{\mathbf{B}}$ & $\alpha_{B}$ & Ik $\left(P_{m}^{B} / \alpha_{B}\right)$ \\
\hline \multicolumn{6}{|l|}{ Station 1} \\
\hline Jul-14 & 202 & 1 & 2.470 & 25.97 & 26.4 \\
\hline Aug-14 & 213 & 1 & 0.981 & 12.24 & 22.3 \\
\hline Aug-14 & 213 & 10 & 0.099 & 2.19 & 12.5 \\
\hline Sep-14 & 251 & 1 & 0.620 & 11.03 & 15.6 \\
\hline Sep-14 & 251 & 18 & 0.247 & 2.25 & 30.4 \\
\hline Sep-14 & 263 & 1 & 0.618 & 8.11 & 21.2 \\
\hline Sep-14 & 263 & 20 & 0.514 & 8.51 & 16.8 \\
\hline Sep-14 & 270 & 1 & 0.600 & 28.96 & 5.8 \\
\hline Sep-14 & 270 & 20 & 0.434 & 17.75 & 6.8 \\
\hline \multicolumn{6}{|l|}{ Station 2} \\
\hline Jul-14 & 198 & 1 & 1.101 & 16.33 & 18.7 \\
\hline Jul-14 & 198 & 40 & 0.072 & 0.67 & 29.8 \\
\hline Jul-14 & 208 & 1 & 2.619 & 12.76 & 57.0 \\
\hline Jul-14 & 208 & 15 & 0.798 & 7.43 & 29.8 \\
\hline Aug-14 & 217 & 1 & 0.709 & 13.17 & 15.0 \\
\hline Sep-14 & 249 & 1 & 0.667 & 3.93 & 47.2 \\
\hline Sep-14 & 256 & 1 & 0.556 & 6.39 & 24.2 \\
\hline Sep-14 & 256 & 17 & 0.448 & 9.15 & 13.6 \\
\hline Sep-14 & 270 & 1 & 0.083 & 1.00 & 23.0 \\
\hline Sep-14 & 270 & 17 & 1.515 & 16.92 & 24.9 \\
\hline \multicolumn{6}{|l|}{ Station 3} \\
\hline Jul-14 & 192 & 1 & 0.779 & 16.30 & 13.3 \\
\hline Jul-14 & 192 & 5 & 2.269 & 47.49 & 13.3 \\
\hline Jul-14 & 200 & 1 & 0.654 & 3.08 & 59.0 \\
\hline Jul-14 & 211 & 1 & 0.573 & 3.52 & 45.2 \\
\hline Jul-14 & 211 & 30 & 0.222 & 2.66 & 23.2 \\
\hline Aug-14 & 219 & 1 & 0.610 & 7.31 & 23.2 \\
\hline Aug-14 & 219 & 26 & 0.155 & 2.86 & 15.0 \\
\hline Sep-14 & 247 & 1 & 0.508 & 9.12 & 15.5 \\
\hline Sep-14 & 247 & 12 & 0.389 & 4.52 & 23.9 \\
\hline Sep-14 & 259 & 1 & 0.598 & 4.60 & 36.0 \\
\hline Sep-14 & 259 & 10 & 0.575 & 2.37 & 67.3 \\
\hline Sep-14 & 271 & 1 & 1.141 & 5.88 & 54.0 \\
\hline Sep-14 & 271 & 10 & 0.616 & 4.59 & 37.3 \\
\hline Oct-14 & 277 & 1 & 0.601 & 7.16 & 23.3 \\
\hline Oct-14 & 277 & 20 & 0.497 & 10.75 & 12.9 \\
\hline Oct-14 & 279 & 1 & 0.567 & 6.82 & 23.1 \\
\hline \multicolumn{6}{|l|}{ Station 4} \\
\hline Jul-14 & 205 & 1 & 0.521 & 2.29 & 63.1 \\
\hline Aug-14 & 215 & 1 & 0.590 & 4.87 & 33.6 \\
\hline Aug-14 & 215 & 30 & 0.398 & 6.48 & 17.1 \\
\hline Aug-14 & 222 & 1 & 0.807 & 13.16 & 17.0 \\
\hline Aug-14 & 222 & 24 & 0.182 & 1.38 & 36.7 \\
\hline Sep-14 & 254 & 1 & 0.417 & 5.12 & 22.6 \\
\hline Sep-14 & 254 & 25 & 0.229 & 3.45 & 18.5 \\
\hline Sep-14 & 261 & 1 & 0.288 & 2.91 & 27.5 \\
\hline Sep-14 & 261 & 30 & 0.152 & 2.37 & 17.9 \\
\hline Oct-14 & 275 & 1 & 0.550 & 6.09 & 25.1 \\
\hline Oct-14 & 275 & 20 & 0.429 & 9.27 & 12.8 \\
\hline
\end{tabular}


Figure S1. Nutrient ratios at all stations and depths sampled. Nitrate + nitrite $(\mu \mathrm{M})$ versus phosphate $(\mu \mathrm{M})$ concentrations (red circles), and nitrate + nitrite $(\mu \mathrm{M})$ v. silicate $(\mu \mathrm{M})$ concentrations (blue squares). Open symbols indicate depths above the average nitracline of the data set $(29 \mathrm{~m})$. Red and blue lines represent the $16 \mathrm{~N}: 1 \mathrm{P}$ and $15 \mathrm{Si}: 16 \mathrm{~N}$ Redfield ratios for phosphate and silicate respectively.

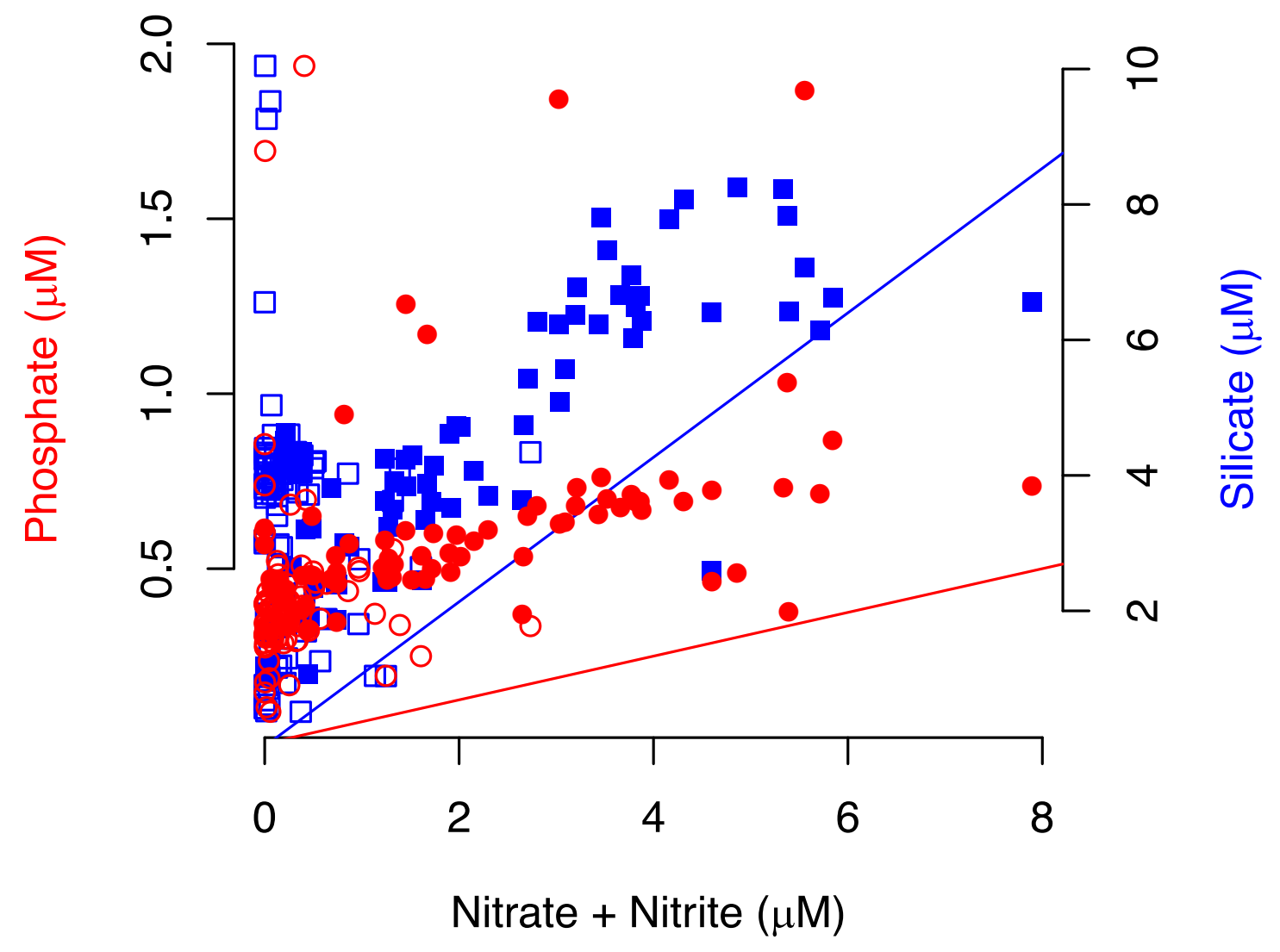


Figure S2. Vertical distribution of chlorophyll a profiles overtime for each station. Colors in legend indicate julian day for each profile.

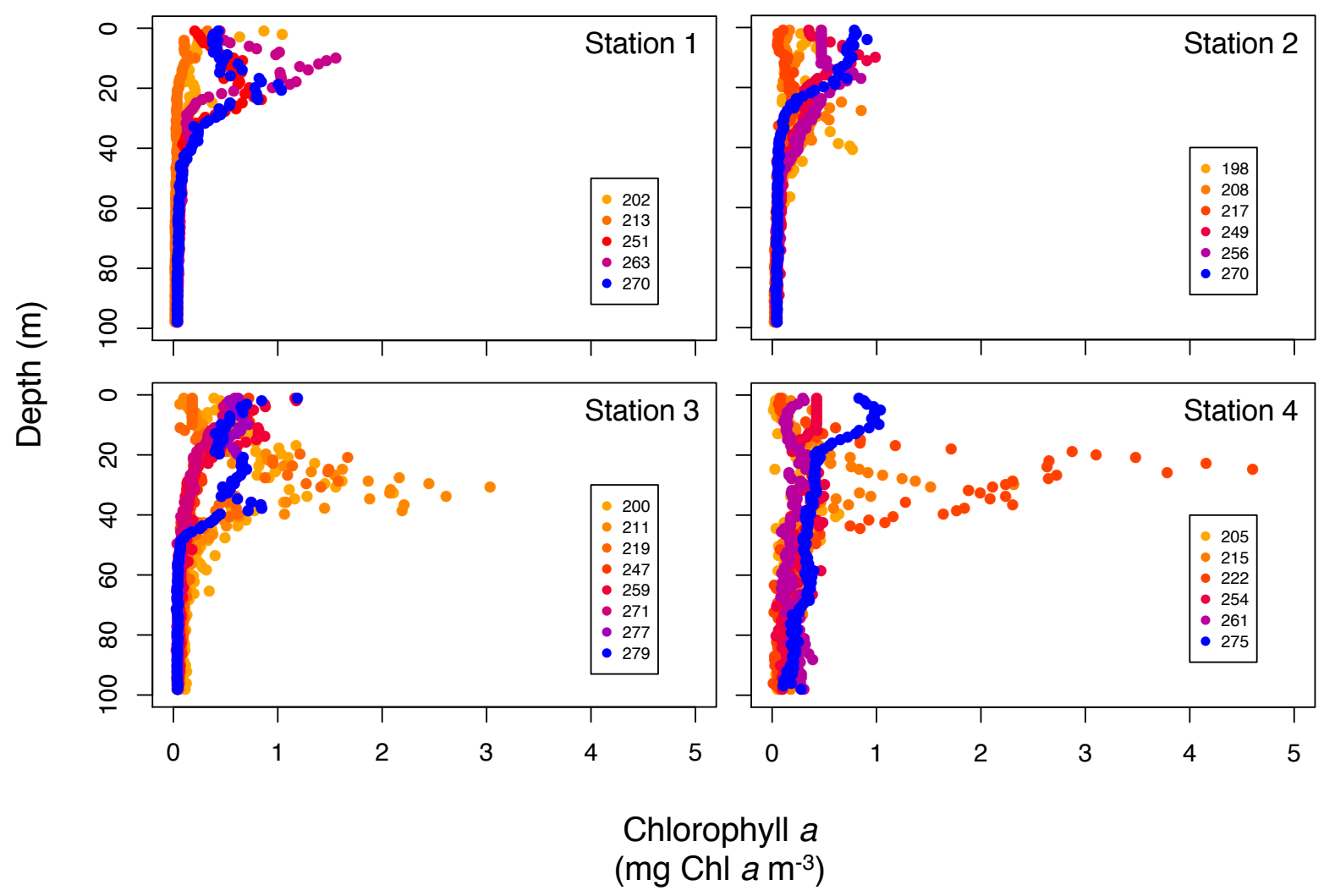


Figure S3. Vertical distribution of primary production profiles overtime for each station. Colors in legend indicate julian day for each profile.

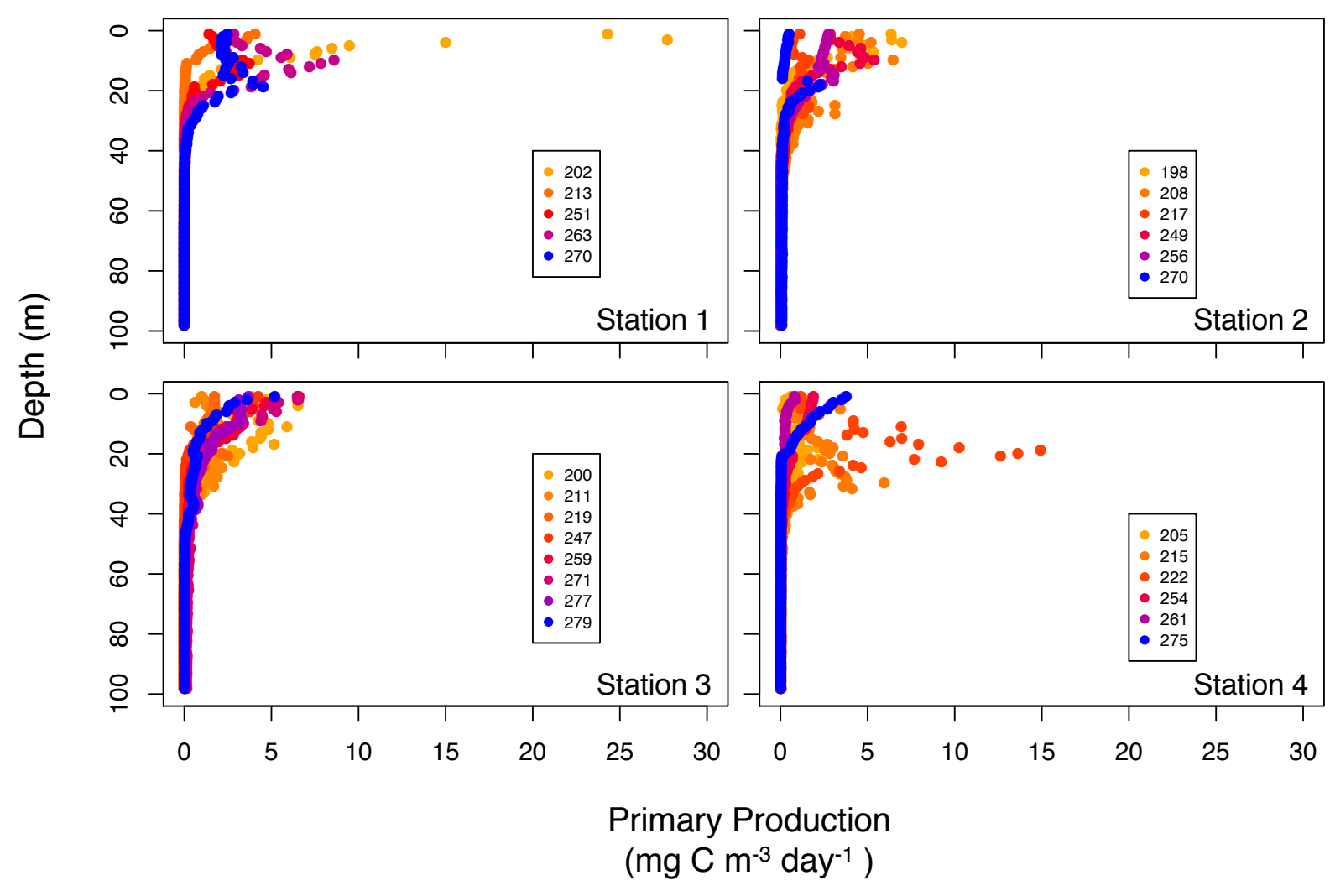

